\title{
Die Studien des Instituts für Technikfolgenabschätzung und Systemanalyse (ITAS) verlegt bei edition sigma
}

\section{Reihe Global zukunftsfähige Entwicklung - Perspektiven für Deutschland}

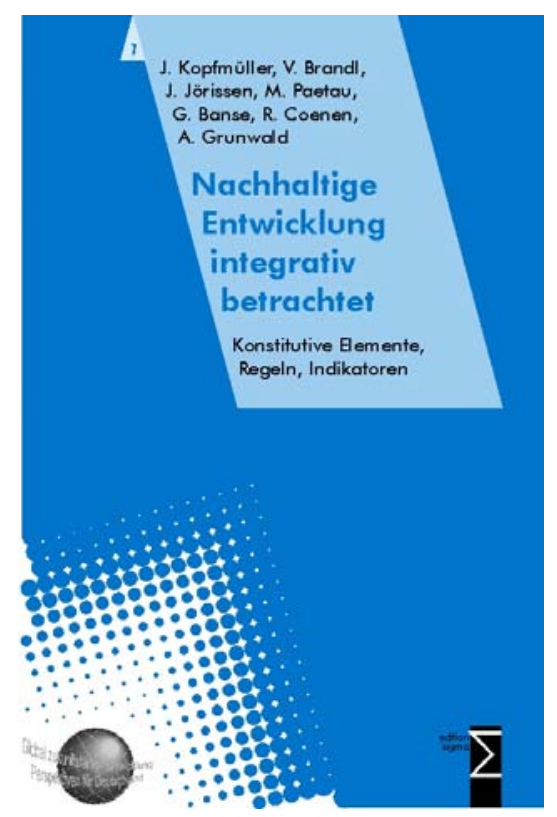

Die Herausforderung einer nachhaltigen Entwicklung ist zu einem beherrschenden Thema in Wissenschaft und Politik geworden. Auch wenn das Leitbild der Nachhaltigkeit weitgehend anerkannt ist, so gehen doch die Vorstellungen darüber weit auseinander, was dies jeweils für konkrete Ziele, Strategien oder Handlungsprioritäten heißt. In diesem Buch wird ein integratives Konzept der Nachhaltigkeit vorgestellt, das seinen Ausgangspunkt in einer gleichrangigen Betrachtung inter- und intragenerativer Gerechtigkeit nimmt. Über Zukunftsfähigkeit wird nicht alternativ in der ökologischen, ökonomischen oder sozialen Dimension von Nachhaltigkeit entschieden, sondern in einer komplexen Verknüpfung zwischen ihnen. Hierbei ergibt sich die Notwendigkeit einer expliziten Berücksichtigung der politisch-institutionellen Dimension. Die Autoren präsentieren ein in sich geschlossenes Konzept, das sich in seiner Sichtweise und Interpretation des Leitbilds von vorhandenen Ansätzen deutlich unterscheidet und diese weiterentwickelt. Das Konzept erstreckt sich auf das Grundgerüst einer integrativen Operationalisierung, auf die Formulierung und Begründung eines Systems von Nachhaltigkeitsregeln und auf dessen Operationalisierung in Form von Indikatoren.

2001, 432 S., ISBN 3-89404-571-X, Euro 25,90

Die internationale Staatengemeinschaft hat sich zwar verpflichtet, das Leitbild der nachhaltigen Entwicklung in konkrete Politik umzusetzen, doch erwartungsgemäß gehen die Vorstellungen über die richtigen Schritte und ihre Abfolge noch weit auseinander. Das interdisziplinäre Verbundprojekt „Global zukunftsfähige Entwicklung - Perspektiven für Deutschland" hat sich zum Ziel gesetzt, begründete Vorschläge für eine Klärung strittiger Fragen vorzulegen. Erste Resultate präsentiert dieser Band im Sinne eines Werkstattberichts: Er enthält Analysen zur Interpretation und Umsetzung des Leitbilds in Wissenschaft und Politik, zu einem integrativen Konzept von Nachhaltigkeit, zu Nachhaltigkeitsindikatoren und gesellschaftlichen Rahmenszenarien. Wesentliche methodisch-konzeptionelle Ansätze des Vorhabens - der Aktivitätsfelderansatz und interaktive Simulationsverfahren - und Ergebnisse zu defizitären Aktivitäts- bzw. Bedürfnisfeldern - Mobilität und Verkehr, Wohnen und Bauen, Ernährung und Landwirtschaft - werden vorgestellt. Darüber hinaus enthält der Band Untersuchungen zu Nachhaltigkeitspotentialen neuer Technologien sowie eine Fallstudie zur Nachhaltigkeit in ländlichen Räumen.

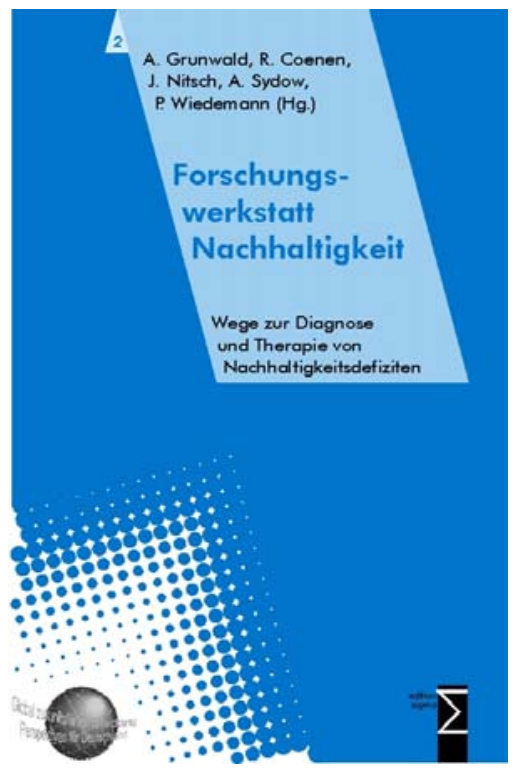

2001, 410 S., ISBN 3-89404-572-8, Euro 24,90 


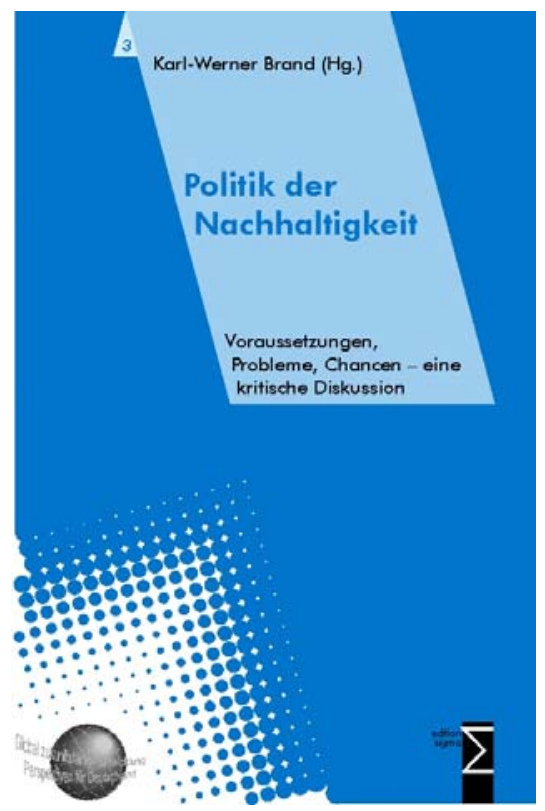

Wiewohl inzwischen eine unübersehbare Fülle an Initiativen, Modellen und Innovationen auf lokaler, regionaler, nationaler und internationaler Ebene im Zeichen des Leitbilds nachhaltiger Entwicklung steht, hat sich am grundlegenden Spannungsverhältnis noch wenig geändert: Das Leitbild als solches ist wenig kontrovers - um so mehr hingegen die Umsetzung. Dieser Diskussionsband versucht eine kritische Bestandsaufnahme der institutionellen Voraussetzungen, Hemmnisse und Chancen des geforderten gesellschaftlichen Transformationsprozesses in Richtung Nachhaltigkeit. Im Mittelpunkt des Bandes steht eine vom BMBF geförderte Sondierungsstudie für die Bundesrepublik. Sie kommt zu einer eher skeptischen Einschätzung der Möglichkeiten einer koordinierten Steuerung komplexer gesellschaftlicher Prozesse und Akteursnetzwerke. Diese Problemdiagnose wird sodann von Experten aus Politik-, Sozial-, Wirtschafts-, Planungsund Rechtswissenschaften kritisch kommentiert. Dabei werden nicht nur zentrale Probleme einer Politik der Nachhaltigkeit sichtbar, sondern es wird auch deutlich, dass die Chancen einer erfolgreichen Bearbeitung dieser Probleme - je nach theoretischer und politischer Position - sehr unterschiedlich gedeutet werden.

2001, 229 S., ISBN 3-89404-573-6, Euro 17,90

Technik entscheidet maßgeblich darüber mit, wie zukunftsfähig unsere Wirtschaftsweise ist. Zukünftige Generationen werden die von unserer Technik produzierten Emissionen und Abfälle sowie die leergeräumten Rohstofflager vorfinden - aber auch den erreichten Wohlstand, die durch Technik ermöglichte Unabhängigkeit von der Natur und vielfältiges durch Technik ermöglichtes Wissen. Das Verhältnis von Technik und Nachhaltigkeit ist also ambivalent. Die Autoren dieses Bandes verbindet die Überzeugung, dass durch innovative Technik und die entsprechende Gestaltung der die Technikentwicklung begleitenden Rahmenbedingungen noch erhebliche Potenziale für den Weg hin zu mehr Nachhaltigkeit mobilisiert werden können. Erforderlich ist - so argumentieren sie -, adäquate Verfahren der Nachhaltigkeitsbewertung, systemanalytische Querschnittsbetrachtungen und konstruktive Technikentwicklung in kohärentem Zusammenklang und gegenseitiger Kooperation zu betreiben. Indem dieses Buch den aktuellen Diskussionstand zum Verhältnis von Technik und Nachhaltigkeit darstellt und zukünftige Perspektiven aufzeigt, wendet es sich gleichermaßen an Forscher und Entwickler wie an Entscheidungsträger in Politik und Wirtschaft.

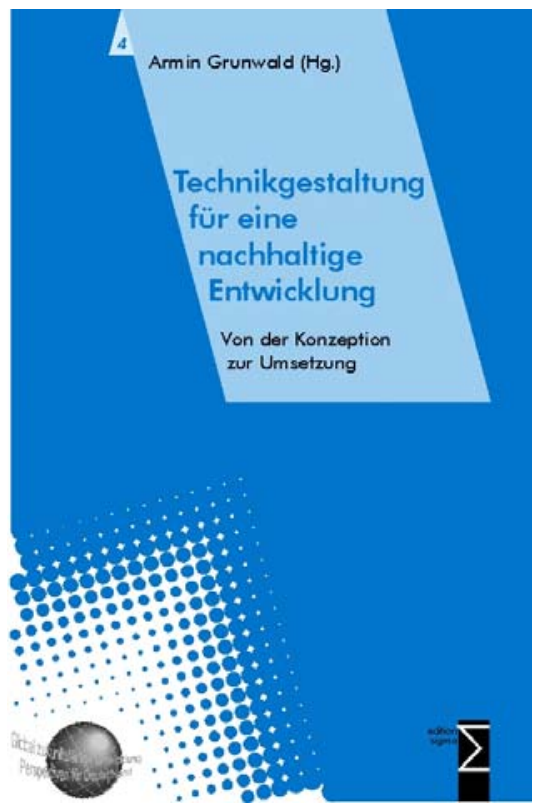

2002, 420 S., ISBN 3-89404-574-4, Euro 25,90 


\section{Reihe Gesellschaft - Technik - Umwelt, Neue Folge}

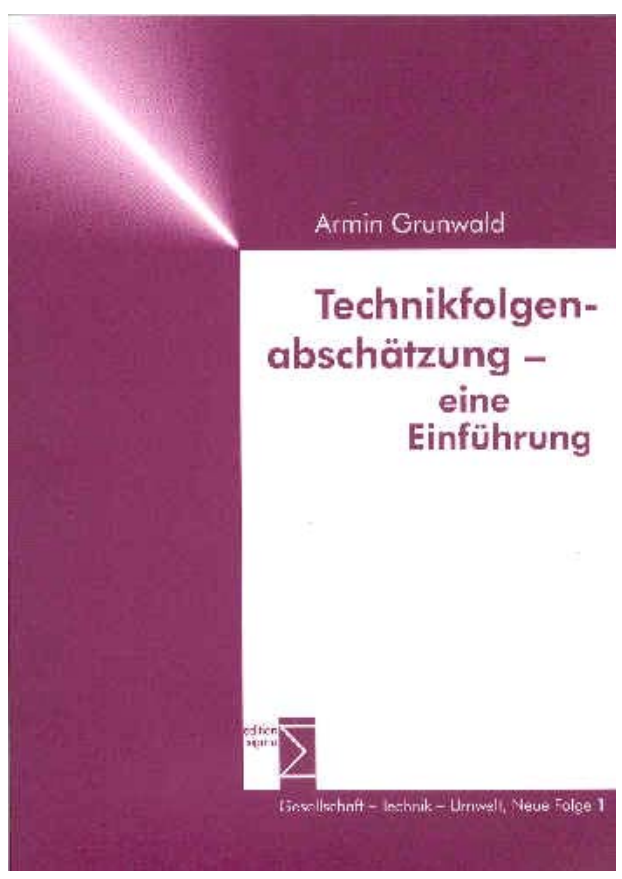

Technikfolgenabschätzung (TA) ist entstanden, als die Nebenfolgen moderner Technik wie technische Risiken, Umwelt- und Akzeptanzprobleme ins gesellschaftliche Bewusstsein traten. Trotz einer über 30jährigen Entwicklung und der vielfältigen Nutzung von TA in der Politikberatung, in Unternehmen und öffentlichen Debatten liegt bislang keine übersichtliche und kompakte Gesamtdarstellung vor. Grunwald schließt nun diese Lücke. Seine Einführung stellt die Frage nach dem gesellschaftlichen Bedarf an den Anfang: Worauf soll TA eine Antwort geben? Ausgehend von realen gesellschaftlichen Problemen mit Technik, Technikfolgen und Technisierung stellt der Autor dar, was TA zur Lösung dieser Probleme beitragen kann, welche Möglichkeiten der Wissenschaften und des politischen Systems genutzt werden können, welche Konzepte und welche Methoden bisher für welche Zwecke entwickelt und eingesetzt wurden, welche Institutionen sich mit TA befassen und wo die Grenzen dieser Vorgehensweisen liegen. Das Buch liefert damit einen orientierenden, verständlichen und dabei doch fachlich avancierten Überblick für alle, die mit Technikfolgen konfrontiert sein können: politische Entscheider, Vertreter aus Wirtschaft, Verbänden und Medien, Wissenschaftler und allgemein an den sozialen Dimensionen der Technikentwicklung Interessierte.

2002, 391 S., ISBN 3-89404-931-6, Euro 24,90

Die Durchdringung aller Lebens- und Arbeitsbereiche mit elektronischen Technologien ist bereits weit fortgeschritten, und es ist gewiss, dass dieser Prozess noch lange nicht zum Stillstand kommt. Er hat mit der breiten Nutzung des Internets sogar nochmals eine neue Qualität erreicht, die die entwickelten Industriegesellschaften in einem grundlegenden Sinn verändert: eine „e-Society“ formiert sich - eine Gesellschaft, die durch Informations- und Kommunikationstechnologien neue Potenziale erschließt, aber auch in wachsende Abhängigkeiten gerät und neuartige Probleme bewältigen muss. In diesem Sammelband, der auf den gleichnamigen Berliner Kongress im Oktober 2001 zurückgeht, werden potenzielle Folgen und Implikationen dieses Prozesses in ihrer politischen, ökonomischen, sozialen und kulturellen Ausprägung ausgelotet. Die Beiträge thematisieren institutionelle Voraussetzungen und Rahmenbedingungen, die für eine zukünftige „e-Society“ erforderlich oder wünschenswert sind. Sie zeigen Gestaltungsmöglichkeiten sowie Handlungs- und Entscheidungsoptionen in einer breiten Palette von Feldern auf; das Spektrum reicht von Electronic Governance über den Zusammenhang von Internet und Kultur bis zu Veränderungen der Arbeitswelt und der Verletzlichkeit der Informationsgesellschaft.

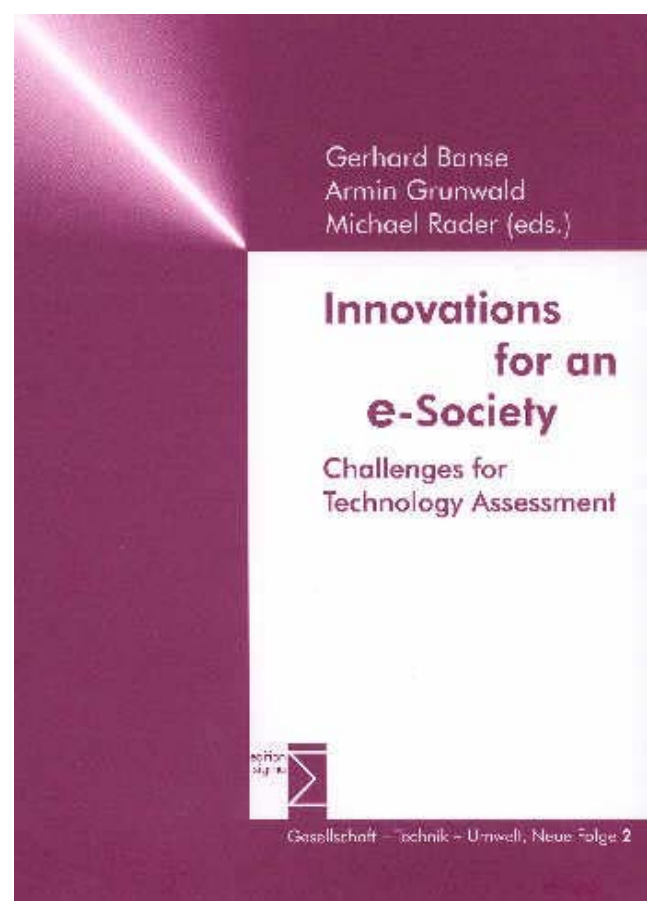




\section{Bestellung}

\begin{tabular}{|c|c|c|}
\hline \multirow[t]{2}{*}{ Anzahl } & \multicolumn{2}{|c|}{$\begin{array}{l}\text { Ich bestelle aus der Reihe „Global zukunftsfähige Entwicklung-Perspektiven für } \\
\text { Deutschland }\end{array}$} \\
\hline & $\begin{array}{l}\text { Bd. 1: Nachhaltige Entwicklung integrativ betrachtet. Konstitutive } \\
\text { Elemente, Regeln, Indikatoren }\end{array}$ & Euro 25,90 \\
\hline & $\begin{array}{l}\text { Bd. 2: Forschungswerkstatt Nachhaltigkeit. Wege zur Diagnose und } \\
\text { Therapie von Nachhaltigkeitsdefiziten }\end{array}$ & Euro 24,90 \\
\hline & $\begin{array}{l}\text { Bd. 3: Politik der Nachhaltigkeit. Voraussetzungen, Probleme, Chan- } \\
\text { cen - eine kritische Diskussion }\end{array}$ & Euro 17,90 \\
\hline & $\begin{array}{l}\text { Bd. 4: Technikgestaltung für eine nachhaltige Entwicklung. Von der } \\
\text { Konzeption zur Umsetzung }\end{array}$ & Euro 25,90 \\
\hline Anzahl & \multicolumn{2}{|l|}{ Ich bestelle aus der Reihe „Gesellschaft-Technik-Umwelt, Neue Folge“ } \\
\hline & Bd. 1: Technikfolgenabschätzung - eine Einführung & Euro 18,90 \\
\hline & $\begin{array}{l}\text { Bd. 2: Innovations for an e-Society. Challenges for Technology As- } \\
\text { sessment }\end{array}$ & Euro 22,90 \\
\hline \multicolumn{3}{|c|}{$\begin{array}{l}\square \text { Ich wünsche kostenlos und unverbindlich weitere Informationen über die Reihe und über } \\
\text { das sozialwissenschaftliche Buchprogramm bei edition sigma. }\end{array}$} \\
\hline \multicolumn{3}{|c|}{ Name, Anschrift: } \\
\hline \multicolumn{3}{|c|}{ Datum, Unterschrift: } \\
\hline
\end{tabular}

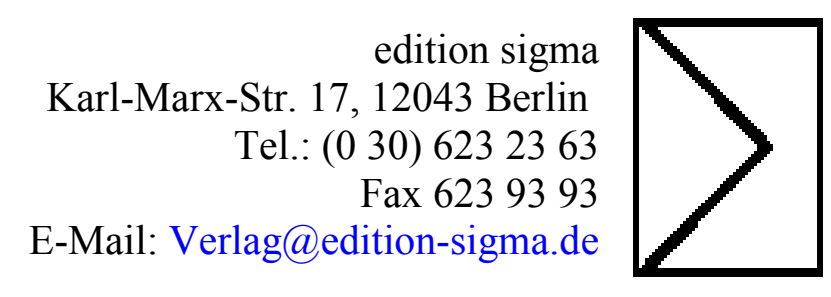

Ständig aktuelle Programminformationen finden Sie jederzeit im Internet:

http://www.edition-sigma.de 